\title{
Compact OCDMA Encoders Based on the Antisymmetric Waveguide Bragg Grating
}

\author{
Jose M. Castro, Student Member, IEEE, Ivan Djordjevic, Member, IEEE, Lyubo Minkov, \\ Christoph M. Greiner, Member, IEEE, Dmitri Iazikov, Member, IEEE, Thomas W. Mossberg, Member, IEEE, and \\ David F. Geraghty, Member, IEEE
}

\begin{abstract}
Optical code-division multiple-access (OCDMA) networks require simple encoders/decoders enabling the integration of optical devices for further development. In this letter, we use silica-on-silicon antisymmetric waveguide Bragg grating devices to demonstrate compact and versatile OCDMA encoder/decoders. These devices use mode conversion to perform all-optical functions on chip.
\end{abstract}

Index Terms-Code-division multiple-access, gratings, integrated optics, optical communication.

\section{INTRODUCTION}

O PTICAL code-division multiple-access (OCDMA) techniques were proposed for optical fiber networks in the late 1980s [1] to provide a more efficient use of the spectral bandwidth, mitigate contention, and provide flexible provisioning. Each user is assigned a unique signature sequence (code), spread in time and/or frequency, designed to minimize the multiuser interference and to maximize the spectrum utilization. Different types of OCDMA have been experimentally demonstrated using fiber Bragg gratings (FBGs) with optical circulators [2] and integrated optics [3]. Providing that each user has the knowledge of a complete set of codewords, OCDMA networks can be designed with fewer hops and tuning delays, and with fast network reconfiguration. By using bulky elements such as FBGs with circulators or arrayed-waveguide gratings, the size and complexity required for each user's encoder/decoder could be prohibitively high even for small networks.

In this letter, we propose and demonstrate a novel OCDMA encoder/decoder based on the antisymmetric waveguide Bragg grating [4]. This type of grating, recently demonstrated for optical add-drop multiplexers [5], eliminates the need for external circulators and allows all optical functions to be performed on the chip. For demonstration purposes, two encoders and one decoder were fabricated using a silica-on-silicon technology [6]. The codes implemented correspond to the family of unipolar balanced incomplete block designs (BIBDs) [7]. These novel encoders would allow the users to have the complete set of codewords in a small integrated chip. This can be used for simulta-

Manuscript received December 13, 2005; revised January 31, 2006. This work was supported by the National Science Foundation (NSF) under Grant ITR 0325979.

J. M. Castro, I. Djordjevic, L. Minkov, and D. F. Geraghty are with Department of Electrical and Computer Engineering, The University of Arizona, Tucson, AZ 85721-0104 USA (e-mail: jmcastro@e-mail.arizona.edu).

C. M. Greiner, D. Iazikov, and T. W. Mossberg are with LightSmyth Technologies, Inc., Eugene, OR 97401 USA.

Digital Object Identifier 10.1109/LPT.2006.872308

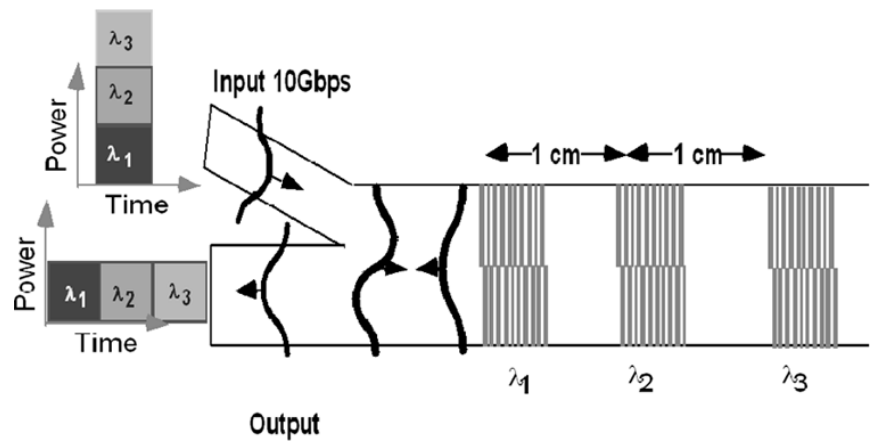

Fig. 1. OCDMA encoder structure schematic and operating principle.

neous decoding by splitting the signal in array of decoders or for fast code reconfiguration using optical switches.

\section{DESIGN AND FABRICATION}

The encoder/decoder structure is shown in Fig. 1. A series of antisymmetric waveguide Bragg gratings are written in a two-mode waveguide. Each grating has a unique periodicity $\left(\Lambda_{i}\right)$ in the longitudinal direction. The gratings are antisymmetric, with a $\Lambda_{i} / 2$ longitudinal shift at the lateral center of the waveguide. Due to this antisymmetry, the gratings convert the fundamental mode (even profile) to the second mode (odd profile) at the Bragg wavelength, $\lambda_{i}=\Lambda_{i}\left(n_{e}+n_{o}\right)$, where $n_{e}$ and $n_{O}$ are the effective indexes of the even and odd modes, respectively. Reflection without mode conversion is not allowed due to the asymmetry of the grating [4], [5]. The precision control required for correct coder diffractive element placement is uniquely enabled by deep ultraviolet photolithographic fabrication, an approach that was recently shown to provide centimeter-scale coherence lengths and nanometer-scale manipulation of individual gating lines [6].

The input and output of the device is through an asymmetric $y$-branch, consisting of two single-mode waveguides of different widths which converge to the two-mode waveguide. Input on the narrower branch will excite the second mode of the two-mode waveguide [4]. The reflections, which have undergone mode conversion [4], will be output on the wider waveguide. This eliminates the need for an external circulator to separate the input form the reflected signal.

Two different encoders (E1 and E2) and one decoder (D1 matched to E1) were fabricated using a silica-on-silicon waveguide technology previously described [6]. The codewords employed were $\lambda_{1} \lambda_{2} \lambda_{3}$ and $\lambda_{2} \lambda_{4} \lambda_{5}$ for E1 and E2, respectively. 


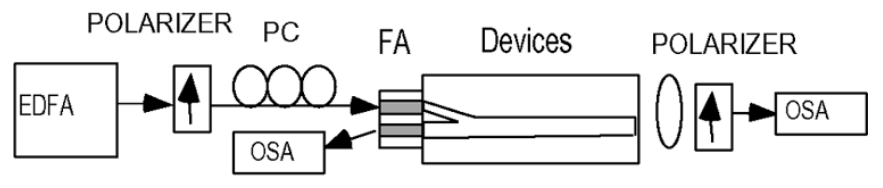

a)

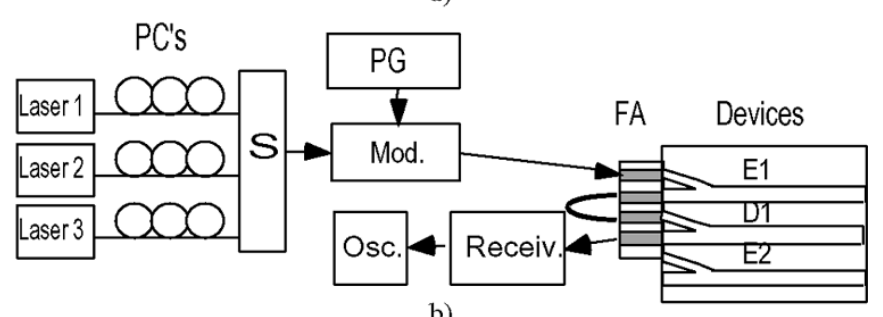

b)

Fig. 2. (a) Setup for transmission and reflection measurements. (b) Setup for encoding/decoding operation.

These are taken from a BIBD set using seven wavelengths and three temporal chips, and serve as a proof of principle [7].

The widths of the branches and waist were 5.6, 8.4, and $14 \mu \mathrm{m}$, respectively. A core structure $2 \mu \mathrm{m}$ thick, with refractive index $n_{\text {core }}=1.46$, was surrounded by a cladding of refractive index $n_{\text {clad }}=1.4457$. Gratings were etched to produce a mesa height of $450 \mathrm{~nm}$, resulting in an index modulation of $\Delta n \sim 4 \times 10-4$. The grating periods required for the design wavelengths were obtained using the effective indexes for the odd and even modes obtained from previous experimental work [5].

The 5.3-mm length of each grating provides $\sim 97 \%$ reflection. The 1-cm separation between adjacent gratings in the fabricated device and the codeword size requires pulse (chip) duration $<=$ 100 ps.

\section{ENCODER/DECODER CHARACTERIZATION}

\section{A. Reflection Spectra}

Fig. 2(a) shows the setup for spectral characterization of the devices. Reflection and transmission spectra were measured using an erbium-doped fiber amplifier (EDFA) as an amplified spontaneous emission (ASE) source and an optical spectrum analyzer with a resolution of $0.06 \mathrm{~nm}$. A polarization controller (PC) allowed characterization of both the TE and TM modes. A fiber array (FA) was used to couple light into and out of the device. After measurements, the spectra were normalized using the EDFAs ASE profile.

Fig. 3 shows the reflection spectrum of the three devices (including coupling and propagation loss). Each device exhibited the three spectral peaks expected from the three gratings. The measured Bragg wavelengths were $\lambda_{1}=1537.40 \mathrm{~nm}$, $\lambda_{2}=1539.13 \mathrm{~nm}, \lambda_{3}=1542.38 \mathrm{~nm}, \lambda_{4}=1540.80 \mathrm{~nm}$, and $\lambda_{5}=1544.14 \mathrm{~nm}$. However, there were small differences between the centers wavelengths of the encoder E1 and decoder D1 ( $\sim .1 \mathrm{~nm}$ on average). Therefore, a perfectly matched filter was not obtained.

A shift in the Bragg wavelength due to the polarization-dependence wavelength was measured as $\sim 0.21 \mathrm{~nm}$. There were not changes in the strength or shape of the reflection of the TE and TM modes. The wavelength shift was produced by the

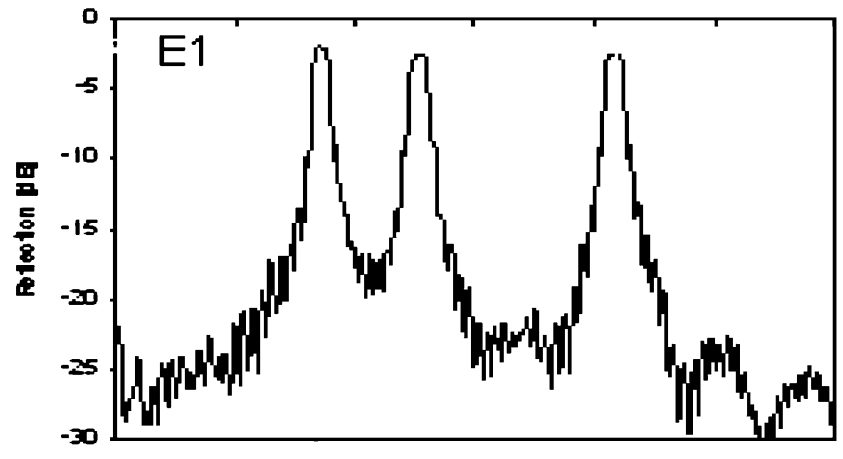

a)

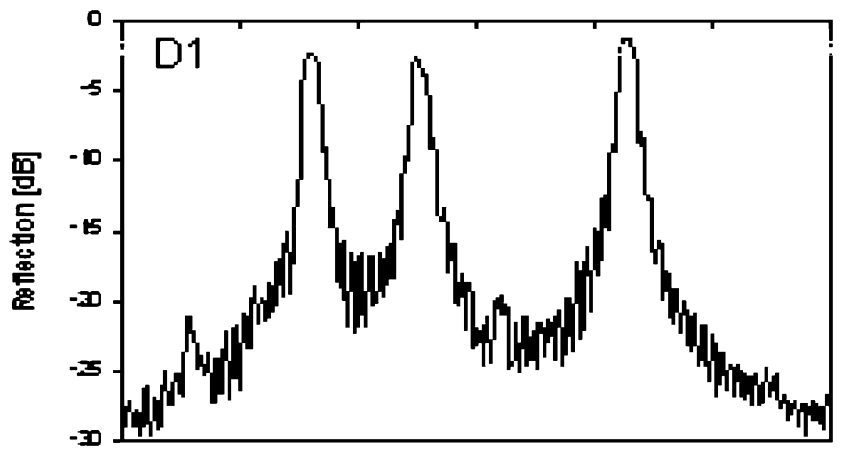

b)

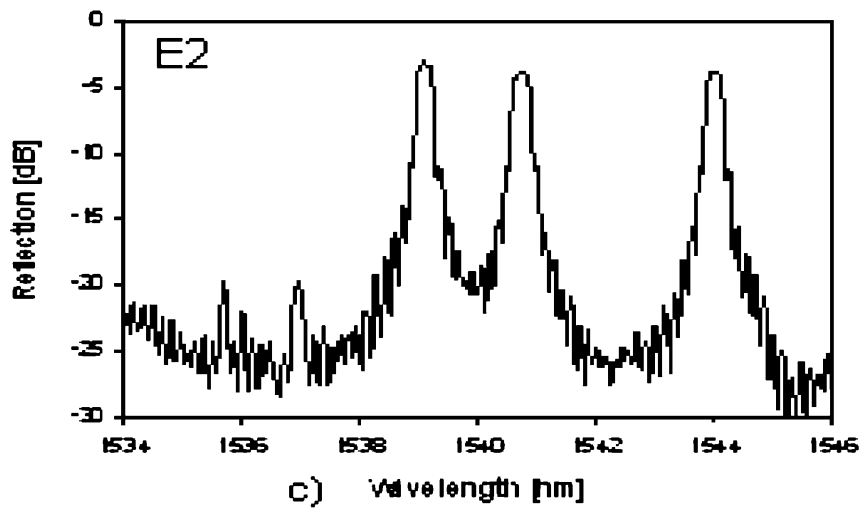

Fig. 3. Measured spectrum: (a) encoder E1; (b) decoder D1; (c) encoder E2.

residual stress and the asymmetry of the waveguide. This can be reduced by techniques described in [8]. Recent results on gratings in dual-layer hybrid waveguide cores have yielded polarization-dependent wavelength shifts as low as $80 \mathrm{pm}[9]$.

\section{B. Encoding/Decoding Operation}

The setup for encoding/decoding demonstration using the proposed devices is shown in Fig. 2(b). It consists of three tunable lasers, PCs, FAs, a wavelength-division multiplexer $(\Sigma)$, a $\mathrm{LiNbO}_{3}$ Mach-Zehnder modulator (Mod), a pattern generator (PG), a receiver, and an oscilloscope (Osc). The laser wavelengths were set to the codeword values, and their combined output was externally modulated with the PG signal. The driver signal for the modulator was nonreturn-to-zero at $10 \mathrm{~Gb} / \mathrm{s}$ with periodic pattern "1000." This is equivalent to a return-to-zero $2.5 \mathrm{~Gb} / \mathrm{s}$ with $25 \%$ duty cycle, as shown in Fig. 4(a). As expected the individual pulses that correspond to $\lambda 1, \lambda 2, \lambda 3$ in the signal to be encoded are aligned in time. 


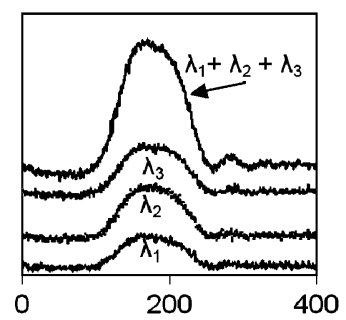

a)

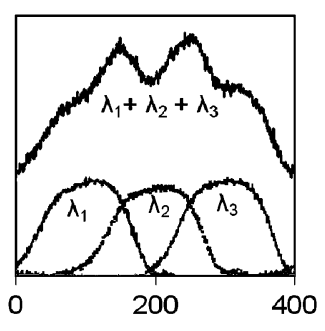

b)
Fig. 4. (a) Input signal to E1; (b) encoded signal from E1.

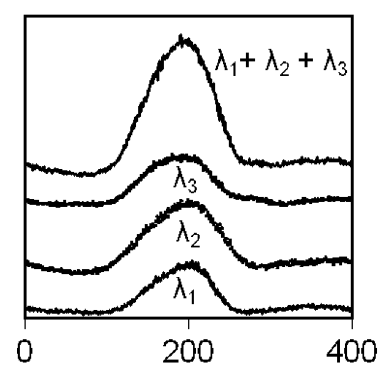

a)

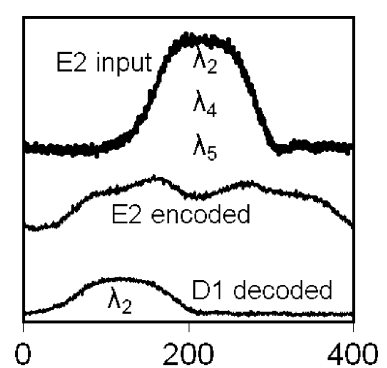

b)
Fig. 5. (a) Output from decoder D1 for input signal encoded with E1 (matched coder and decoder). (b) Input and coded output from coder E2 along with output from decoder D1 (mismatched coder and decoder).

The signal from the modulator is launched to the input of the encoder E1, where the Codeword 1 is imposed. The output of the encoder, as well as its individual spectral components, is shown in Fig. 4(b). The encoded signal has two peaks between time chips due to overlap of individual pulses. The insertion loss of the coding process is $\sim 4 \mathrm{~dB}$.

After amplification by an EDFA, the encoded signal from E1 is sent to the input of the decoder D1 which has the reverse configuration of E1 encoder. The decoder realigns the pulses of each individual wavelength in time, as shown in Fig. 5(a). The source laser powers were adjusted to compensate for unequal EDFA gain. The lack of perfect match in the center wavelengths produces small variations in the contribution from each wavelength. Despite this small imperfection, as demonstrated below, clear distinction between matching and nonmatching decoder inputs is achieved. The pulse with power (the addition is incoherent) equivalent to the sum of the three individual pulses is recovered at the same repetitions rate as that of encoder input.

Additionally, the process of encoding with E2 and decoding with D1 is performed. The encoded signal from E2 is a spread signal that employs $\lambda_{2}, \lambda_{4}, \lambda_{5}$, as shown in Fig. 5(b). The decoded signal with D1 contains only a small fraction the signal incident to E2, since the codewords for E1 and E2 have only one wavelength in common. Insertion loss produced by the decoder was $\sim 4 \mathrm{~dB}$. Insertion losses in the encoder and decoder are caused mainly by butt coupling from the FA to the $\mathrm{y}$-branches. Propagation losses were estimated in $\sim 0.1 \mathrm{~dB} / \mathrm{cm}$.

\section{SUMMARY}

We have designed and experimentally demonstrated OCDMA encoders/decoders based on the novel antisymmetric waveguide Bragg gratings and asymmetric $y$-branches. These devices, fabricated in silica-on-silicon, can be applied to numerous OCDMA coding schemes that at the present requires FBGs and circulators.

Integrated devices in silica-on-silicon would provide significant advantages to OCDMA systems. For example, a small silica-on-silicon chip with the complete set of codes would allow direct communication among all the users and flexible operation. Tunability is not required for these devices. Optical switching among the codes contained in a chip can produce faster network reconfiguration without increasing the complexity or size of the encoders/decoders. The integration with other elements of the system (lasers, detectors) would be a step toward the implementation of small network cards for OCDMA.

\section{REFERENCES}

[1] J. A. Salehi, "Code division multiple-access techniques in optical fiber networks-Part I: Fundamental principles," IEEE Trans. Commun., vol. 37, no. 8, pp. 824-833, Aug. 1989.

[2] X. Wang et al., "Novel temporal/spectral coding technique based on fiber Bragg gratings for fiber optic CDMA application," presented at the Optical Fiber Communication Conf., 1999, and the Int. Conf. Integrated Optics and Optical Fiber Communication (Dig. OFC/IOOC '99), San Diego, CA, Feb. 1999, Paper WM50.

[3] K. Takiguchi, T. Shibata, and M. Itoh, "Encoder/decoder on planar lightwave circuit for time-spreading/wavelength hopping optical CDMA," Electron. Lett., vol. 38, pp. 469-470, May 2002.

[4] J. M. Castro, D. F. Geraghty, S. Honkanen, C. M. Greiner, D. Iazikov, and T. W. Mossberg, "Demonstration of mode conversion using anti-symmetric waveguide Bragg gratings," Opt. Express, vol. 13, pp. 4180-4184, May 2005.

[5] J. M. Castro, D. F. Geragthy, S. Honkanen, C. Greiner, D. Iazikov, and T. W. Mossberg, "Optical add-drop multiplexers based on the anti-symmetric waveguide Bragg grating," Appl. Opt., to be published.

[6] C. Greiner, T. W. Mossberg, and D. Iazikov, "Bandpass engineering of lithographically scribed channel-waveguide Bragg gratings," Opt. Lett., vol. 29, pp. 806-808, 2004.

[7] I. B. Djordjevic and B. Vasic, "Novel combinatorial constructions of optical orthogonal codes for incoherent optical CDMA systems," J. Lightw. Technol., vol. 21, no. 9, pp. 1859-1875, Sep. 2003.

[8] R. Adar, C. H. Henry, R. H. Kistler, and R. F. Kazarinov, "Polarization independent narrow band Bragg reflection gratings made with silica-onsilicon waveguides," Appl. Phys. Lett., vol. 60, pp. 1779-1781, 1992.

[9] C. M. Greiner, D. Iazikov, and T. W. Mossberg, "Low-loss silica-onsilicon two dimensional Fabry-Perot cavity based on holographic Bragg reflectors," Opt. Lett., vol. 30, no. 1, pp. 38-40, 2005. 